\title{
Pensamiento mágico en la psicología: REPRESENTACIONES EN DOCENTES
}

\author{
FABRIzIO LÓPEZ De POMAR \\ Universidad Nacional de Buenos Aires, Argentina \\ Recibido: 12 de julio del 2019 / Aceptado: 10 de setiembre del 2019 \\ doi: 10.26439/persona2019.n022(2).4567
}

\begin{abstract}
Resumen. Las universidades son centros educativos que promueven la formación científica, Diversas investigaciones han cuestionado la presencia del pensamiento mágico en carreras de ciencias, como en el caso de la psicología. Se buscó contribuir en esa línea de trabajo mediante una estrategia distinta de la cuantitativa en estudiantes universitarios de psicología. Bajo un enfoque cualitativo, se analizaron las representaciones de docentes de psicología sobre el pensamiento mágico en la formación y profesión psicológica, Se realizaron entrevistas semiestructuradas y se organizó la información en temas, categorías y subcategorías. La discusión aborda los consensos y disensos hallados en torno al entendimiento que tiene cada docente sobre la ciencia, la psicología, sus alcances y límites.
\end{abstract}

Palabras clave: pensamiento mágico / representaciones docentes / educación científica / psicología

\section{MAGICAL THINKING IN PSYCHOLOGY: PROFESSORS' REPRESENTATIONS}

\begin{abstract}
Universities are education centers that promote scientific training. Several studies have questioned the presence of magical thinking in students attending undergraduate programs in science such as psychology students. Using a strategy different from the quantitative approach in university students of the psychology undergraduate program, this research aimed to contribute to this line of work. Psychology professors' representations of magical thinking in psychology training and career were analyzed using a qualitative approach. Semi-structured interviews were performed, and the collected information was organized in topics, categories and subcategories. The discussion addresses the agreements and disagreements found on professors' understanding of science, psychology, their scope and limits.
\end{abstract}

Keywords: magical thinking / teaching representations / scientific education / psychology 


\section{INTRODUCCIÓN}

Se considera que la educación debe brindar conocimientos científicos del mundo a las personas, de modo que puedan participar en la toma de decisiones políticas para la conservación del equilibrio social y medioambiental, así como fomentar la generación del conocimiento al servicio de la humanidad (Declaración de Budapest, 1999; Unesco, 2016).

Las universidades pertenecen al conjunto de entidades responsables de la formación científica; una prueba de ello se encuentra al revisar sus compromisos institucionales, Otra prueba puede hallarse en la literatura respectiva, al evidenciar el interés a lo largo de las décadas por monitorear el desarrollo del pensamiento científico en universidades, evaluando la presencia de un pensamiento antagónico: el pensamiento mágico, pues se espera que los profesionales de ciencia respondan a incógnitas del mundo aludiendo a explicaciones naturales y no sobrenaturales (Caldera, Reynoso, Zamora y Pérez, 2017; Dudycha, 1933; Jahoda, 1968; Otis y Kuo, 1984; Petra y Estrada, 2014; Sagone y De Caroli, 2015; Salter y Routledge, 1971; Silva Santisteban, 1975; Regal, 1993; Wagner, 1928). El mismo interés se observa en el ámbito de la psicología (Caldera, Amador, Reynoso y Zamora, 2015; Cárdenas, Gallardo, Adaos y Bahamondes, 2013; McCaffree y Saide, 2014; Messer y Griggs, 1989; Tupper y Williams, 1986; Valentine, 1936).

Las conceptualizaciones clásicas del pensamiento mágico lo describen como un razonamiento precientífico que busca explicar y controlar los fenómenos de la naturaleza (internos y externos), asociando inadecuadamente los eventos, animando lo inanimado y antropomorfizando objetos o procesos para dar sentido a lo desconocido (Frazer, 2011; Freud, 1913; Lévi-Strauss, 1997; Malinowski, 1948; Piaget, 1929; Tylor, 1920).

La APA define el pensamiento mágico como la creencia en poder influir sobre la conducta de los demás o en eventos a distancia a través del pensamiento, los deseos o rituales (VandeBos, 2006). Los trabajos de las últimas décadas, que incluyen definiciones ofrecidas por las escalas más usadas de pensamiento mágico, permiten entenderlo como un conocimiento de la realidad que desafía los principios y causalidades básicas establecidos por la ciencia estándar, sosteniendo sus explicaciones en agentes o mecanismos sobrenaturales (Eckblad y Chapman, 1983; Kingdon, Egan y Rees, 2012; Moral, 2009; Tobacyck, 1984).

Se encuentra documentada la preocupación por formar futuros profesionales de psicología que interpreten la realidad conforme a una lógica científica (Caldera et al., 2015; Cárdenas et al., 2013). Dicha preocupación se sostiene en resultados y conclusiones que señalan al pensamiento mágico como participante en el desempeño académico de los estudiantes (Farkas, 2003; Messer y Griggs, 1989; Rudski y Edwards, 2007; Tobacyk, 1984), así como en interpretaciones inadecuadas de conceptos básicos de psicología y en el ejercicio del pensamiento crítico (Bensley, Lilienfeld u Powell, 2014; McCaffree y Saide, 2014). 
Las representaciones son una forma de conocimiento que se comparte consensualmente y se construye mediante la interacción social de un grupo inmerso en una determinada cultura, lo que permite volver lo poco familiar y complejo (como conceptos, procesos o teorías científicas) en familiar y simple, para poder afrontar las actividades cotidianas. Este tipo de conocimiento se construye de una manera informal, es decir, no es producto de una construcción sistemática y rigurosa (Moscovici, 1993). Las representaciones se han estudiado en el ámbito educativo por las implicancias que pueden tener en los procesos de enseñanza-aprendizaje, pues lo que entiendan los docentes sobre determinado objeto o proceso participará en dicha enseñanza y podrá ser interiorizado por los estudiantes (Ávila, 2001; Fraile, Prieto y Bacaicoa, 2005; Covarrubias, 2016; Damn, 2014; Mazzitelli, Aguilar, Guirao y Olivera, 2009; Molina, 2003; Travé, 2013).

La presente investigación se suma a los esfuerzos colectivos por entender la presencia y repercusiones del pensamiento mágico dentro de la ciencia, en este caso, en la formación y profesión de la psicología. Para ello se utilizó una estrategia que, hasta donde se pudo revisar, no ha sido aplicada. Se buscó explorar y describir las representaciones que tienen los docentes de psicología sobre la presencia del pensamiento mágico en la formación de sus estudiantes, así como en la práctica profesional del psicólogo.

\section{MÉTODO}

\section{Diseño de estudio}

Para cumplir con los objetivos, se utilizó un diseño fenomenológico (Castaño y Quecedo, 2002; Flick, 2015) orientado a comprender las experiencias de las personas para descubrir los elementos convergentes y divergentes en sus vivencias, identificando elementos significativos que permitan generar categorías (Starks y Brown, 2007).

\section{Técnica e instrumentos}

La técnica empleada fue la entrevista cualitativa (Denzin y Lincoln, 2012; Robles, 2011). Se recabó información haciendo uso de una guía temática de preguntas y una ficha de datos personales (ver tabla 1). Los ejes temáticos de la entrevista fueron la posible división y jerarquía de las explicaciones naturales y sobrenaturales, la naturaleza y demarcación del pensamiento mágico con conceptos y áreas afines y las posibles implicancias del pensamiento mágico en la formación y profesión psicológica. Todas las preguntas que se formularon para la guía fueron revisadas por un educador, un psicólogo y un filósofo de la ciencia. Así también, la validez de la entrevista cualitativa se sostiene en la libertad que ofrece a los entrevistados para responder de manera crítica a las preguntas y suposiciones del investigador (Kvale, 2011). También se consideró la validez ecológica al realizar las entrevistas en los centros laborales de los docentes participantes (Willig, 
2013). Para el registro de la información, se empleó una grabadora de audio, hojas de papel y lapiceros.

\section{Participantes}

Se seleccionaron docentes de psicología bajo un muestreo intencional, de acuerdo con su interés y disponibilidad en participar sobre el tema, con un criterio no probabilístico y en cadena, lo que culminó al cumplirse el criterio de saturación (Flick, 2015; Hernández, Fernández u Baptista, 2014; Martínez-Salgado, 2011). Los participantes fueron en total once docentes de psicología: seis mujeres y cinco hombres, con edades comprendidas entre 31 y 70 años.

\section{Procedimiento}

El proceso de recolección de datos se realizó entre los meses de noviembre de 2017 y enero de 2018, según la disponibilidad de los participantes. Se realizó la guía de entrevista con los ejes temáticos relacionados al objetivo del estudio y revisados por profesionales en torno a dichas temáticas. Se elaboraron los consentimientos informados y se enviaron correos electrónicos a varios docentes invitándolos a participar. Se realizaron las coordinaciones respectivas para acceder a los participantes, ya sea en su ambiente universitario de trabajo o domicilio, dependiendo siempre de la comodidad del docente,

Tabla 1

Datos personales de los entrevistados $(N=11)$

\begin{tabular}{|c|c|c|c|c|c|}
\hline $\begin{array}{c}\text { Entrevistado } \\
\text { (a) }\end{array}$ & $\begin{array}{l}\text { Rango } \\
\text { de edad } \\
\text { (años) }\end{array}$ & Religión & Grado académico & $\begin{array}{l}\text { Años de } \\
\text { docencia }\end{array}$ & Asignaturas a cargo \\
\hline Mónica & $51-60$ & Ninguna & $\begin{array}{l}\text { PhD Psicología } \\
\text { Clínica }\end{array}$ & 11 & $\begin{array}{l}\text { Desarrollo II; Dis.y eval. de } \\
\text { programas }\end{array}$ \\
\hline David & $31-40$ & Ninguna & Mg. Filosofía & 14 & $\begin{array}{l}\text { Fil. de la ciencia; Análisis } \\
\text { fenomenológico de la } \\
\text { personalidad }\end{array}$ \\
\hline Cristina & $51-60$ & Católica & $\begin{array}{l}\text { Mg. en Antropología } \\
\text { y PhD en Psicología }\end{array}$ & 30 & $\begin{array}{l}\text { Sem. tesis; sem. preliminar } \\
\text { de tesis }\end{array}$ \\
\hline César & $41-50$ & Ninguna & $\begin{array}{l}\text { Licenciado } \\
\text { Psicología clínica }\end{array}$ & 15 & $\begin{array}{l}\text { Estrat. Metacog. del } \\
\text { aprendizaje }\end{array}$ \\
\hline María & $41-50$ & Católica & $\begin{array}{l}\text { Mg. en investigación } \\
\text { y docencia } \\
\text { universitaria }\end{array}$ & 14 & $\begin{array}{l}\text { Psic. comunitaria; psic. } \\
\text { Educacional }\end{array}$ \\
\hline Sara & $61-70$ & Ninguna & $\begin{array}{l}\text { PhD en ciencias } \\
\text { cognitivas }\end{array}$ & 28 & Introducción a la Psicología \\
\hline
\end{tabular}


(continuación)

\begin{tabular}{|c|c|c|c|c|c|}
\hline Ana & $31-40$ & Católica & $\begin{array}{l}\text { Mg. en Psicología } \\
\text { Clínica y de la salud }\end{array}$ & 10 & $\begin{array}{l}\text { Psic. cognitiva conductual y } \\
\text { sistémica; desarrollo del niño } \\
\text { y del adolescente }\end{array}$ \\
\hline Victoria & $61-70$ & Católica & $\begin{array}{l}\text { Mg. en } \\
\text { Administración }\end{array}$ & 32 & Psic. general \\
\hline Guillermo & $51-60$ & Católico & PhD en Psicología & 31 & $\begin{array}{l}\text { Controversias y desafíos en la } \\
\text { educación peruana; asesoría } \\
\text { de tesis }\end{array}$ \\
\hline Sergio & $31-40$ & Ninguna & Mg. en Ética & 15 & $\begin{array}{l}\text { Epistemología; Teoría del } \\
\text { Conocimiento }\end{array}$ \\
\hline Alberto & $61-70$ & Católico & PhD en Ciencias & 30 & Historia de la Psicología \\
\hline
\end{tabular}

Nota: todos los participantes realizaron sus estudios de pregrado en psicología.

Se garantizaron las condiciones ambientales adecuadas para los diferentes lugares en los cuales se recolectó la información. Se brindaron las mismas consignas y se contó con los materiales necesarios. Se respetó la guía temática para la entrevista y en ocasiones se agregaron preguntas para profundizar en puntos que se consideraron relevantes para el propósito de la investigación. Se logró obtener respuestas desde una actitud espontánea y colaboradora. Una vez que se obtuvo la información de los participantes, se transcribieron las entrevistas a formato de texto Word para luego realizar el procesamiento de datos.

\section{Procesamiento de datos}

Se procedió a una primera revisión general de toda la información recabada para contar con un panorama completo. A continuación, se identificaron las unidades de análisis, tomando como criterio de separación la unidad temática, lo que permitió generar categorías. Luego, se relacionaron entre sí las categorías para obtener patrones temáticos. El procesamiento de la información cualitativa se sostuvo en procesos y operaciones lógicas analíticas inductivas (Gibbs, 2012). Finalmente, se desarrolló una narrativa del panorama general, con las categorías y subcategorías emergentes a partir de las representaciones que tenían los participantes.

Para incrementar la rigurosidad metodológica, se tomaron dos acciones: (i) agregar un participante luego de haber cumplido con la saturación de categorías; se hizo para observar si sus respuestas se acoplaban a las categorías anteriores, encontrándose confirmación; (ii) se volvió a entrevistar a tres docentes, con el propósito de obtener su validación sobre lo que se entendió de sus respuestas y para que puntualicen sobre aquello que quedó abierto y proclive a múltiples interpretaciones del investigador. 


\section{RESULTADOS}

El primero de los tres temas exploró en los docentes los tipos de explicaciones que predominan a la hora de responder ante los eventos del mundo, y si acaso esos tipos de explicaciones pueden ordenarse jerárquicamente o no (figura 1).

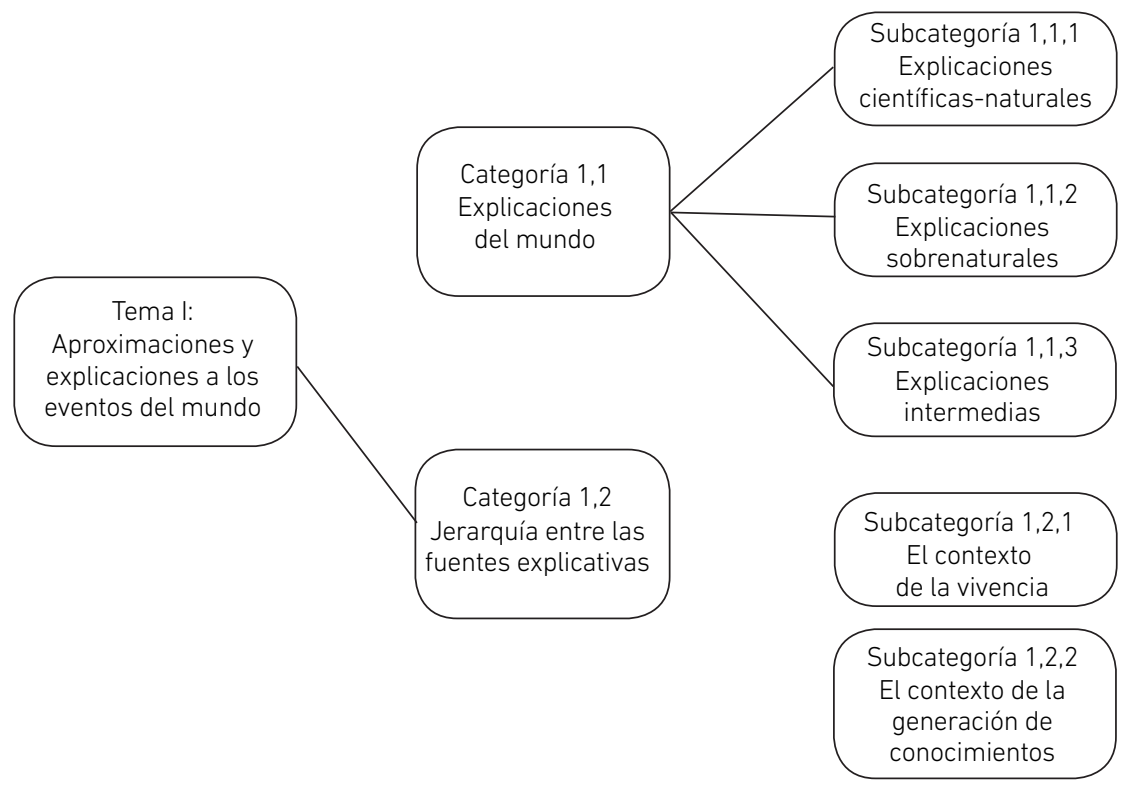

Figura 1. Organización esquemática del primer tema, sus categorías y subcategorías

Se halló en las respuestas que las explicaciones científicas-naturales (términos intercambiables por los mismos docentes) presentan elementos y mecanismos que pueden ser conectados con otros campos de la ciencia. Son producto de un proceso riguroso y crítico. Generan propuestas explicativas que pueden ser contrastadas con otros trabajos y permiten una discusión en aras de un consenso y renovación del conocimiento a la fecha,

Una explicación natural es una explicación que se basa fundamentalmente en el conocimiento científico, que es verificable [...] que es falsable en primer lugar, ¿no es cierto? Que se va guiando y se va modificando según el avance de la ciencia. (Alberto)

Se señaló que las explicaciones sobrenaturales presentan elementos y mecanismos por encima de los postulados naturales, como las divinidades u otras explicaciones difícilmente accesibles o comprobadas por la ciencia, por lo que no gozan de mucha credibilidad, como señalan los docentes. Están muy vinculadas con lo desconocido, con lo inexplicable: 
Va más allá de nuestra comprensión, de nuestra elaboración, de nuestra categorización, de nuestro procesamiento incluso científico y desde esa perspectiva se convierte en algo que va más allá de lo natural... (Cristina)

Una de las explicaciones intermedias (por no estar caracterizadas completamente dentro de las dos anteriores) es la explicación pseudocientífica, la cual no necesariamente presenta elementos sobrenaturales, sino más bien una errónea causalidad cuando asocian eventos. La otra, un dualismo naturalista, como señala el siguiente docente:

[...] no todas las explicaciones científicas tienen que ser materialistas necesariamente [...] la información no es material, ¿qué es la información? La información es básicamente un espacio de posibilidades [...] Ahora, la información parece que es una propiedad fundamental del universo. Y no es material. (David)

Con respecto a la posible jerarquía entre las explicaciones, los docentes la consideraron circunstancial. Si se trataba del ámbito íntimo, no había jerarquía, pues importa la atribución que haga cada persona y encaje de acuerdo a su sistema de creencias. La coherencia es lo que brinda sentido y eso genera un equilibrio interno:

[...] no creo que necesariamente tenga que ser algo jerárquico, yo creo que son dos cosas separadas pero que tienen, digamos, funciones diferentes o propósitos diferentes, que se pueden integrar [...]. (Mónica)

Es que yo creo que tiene que ver con cuánto te dice la ciencia y cuánto te dice otra postura, con respecto al fenómeno. Porque finalmente uno trata de descubrir cuál es el significado de la realidad. (Cristina)

Cuando se trata de generar conocimiento que permita desarrollar tecnología y técnicas de intervención para mejorar la calidad de vida de las personas, se encontró que los docentes señalaban una jerarquía, una verticalidad.

En mi fuero interno sí creo que el conocimiento científico es superior a muchas cosas. ¿Por qué? Por los resultados. En todo, en todas las ramas. A la hora que una rama del conocimiento se hace científica, comienza a producir frutos [...]. Entonces por eso yo creo que hay que darle prioridad a este tipo de conocimiento. (Sergio)

Pero eso de horizontalidad es solamente para el proceso de curación, no es para la ciencia, porque la ciencia sí es otra categorización. Ahí sí hay verticalidad. (Sara)

El segundo de los tres temas exploró las representaciones en torno al concepto y áreas lindantes al pensamiento mágico (figura 2).

Según las representaciones que tienen los docentes sobre el pensamiento mágico, es posible presentarlo como un proceso de interpretación de la realidad que hace uso de elementos que no concuerdan con las explicaciones científicas, naturales. Las explicaciones generadas por el pensamiento mágico son producto de elementos predominantemente afectivos, por lo tanto, son irreflexivas. Movilizadas por los deseos y temores, las respuestas del pensamiento mágico buscan certezas que brinden control y 
significado a la vida de quien las sostiene. Al tener ese objetivo, el pensamiento mágico genera respuestas que no provienen de un proceso riguroso donde se contrasten con otras posibles explicaciones.

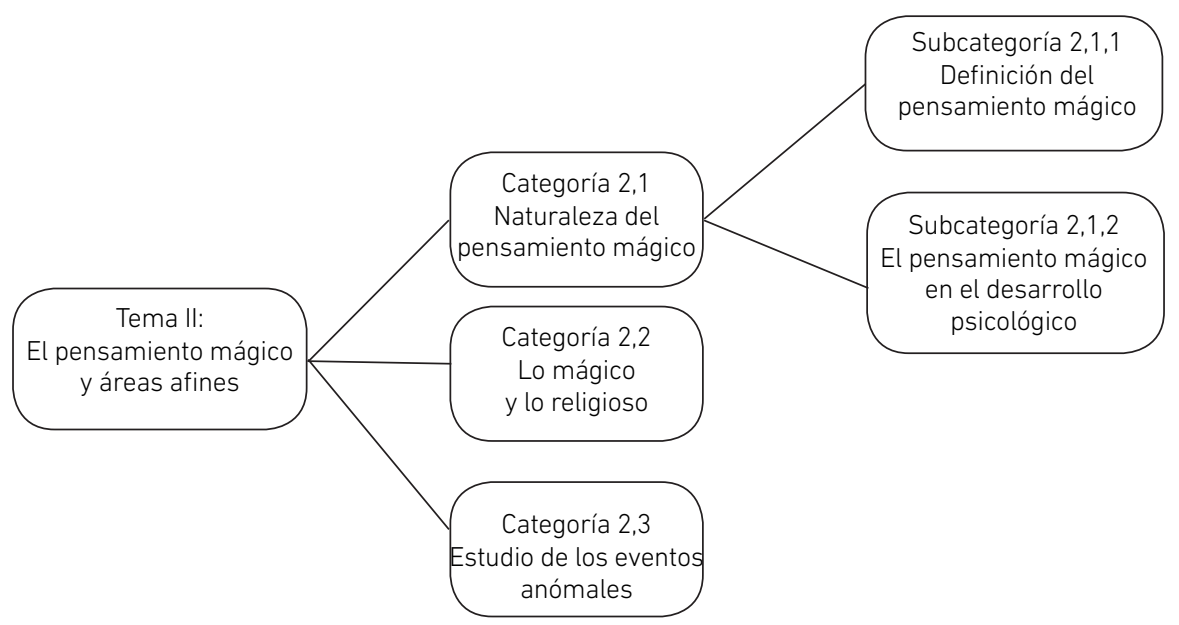

Figura 2. Organización esquemática del segundo tema, sus categorías y subcategorías

El pensamiento mágico es básicamente un pensamiento que interpreta la realidad a partir de causas y efectos que no necesariamente tienen una conexión real, [...] es una relación arbitraria entre las causas y los efectos. (César)

El pensamiento mágico es un pensamiento afectivo [...] y siendo eso, es tremendamente especulativo, descontrolado. (David)

Con respecto al desarrollo del pensamiento mágico, los participantes lo consideran como algo connatural al ser humano. Se observa en infantes como parte de las etapas del desarrollo psicológico. Asimismo, conforme se va desarrollando la persona, el grado en el cual se manifiesta el pensamiento mágico es variable, pero sigue presente. En algunos casos, el pensamiento mágico fue señalado como parte de la evolución del pensamiento, y conforme se adquiere mayor madurez psicológica, menor será la presencia del pensamiento mágico.

[...] un máximo nivel de desarrollo y si quieres madurez, está asociado a flexibilidad cognitiva, etc., y esto se articula con un máximo nivel de desarrollo afectivo y moral. Entonces yo creo que ese óptimo funcionamiento afectivo, moral y cognitivo te permiten un desarrollo no mágico. (Cristina)

Con respecto a las diferencias o similitudes entre lo mágico y lo religioso, se encontraron dos posiciones contrapuestas. Aquellos docentes que diferencian el pensamiento religioso del mágico, lo hacen pues conciben a este último como el generador 
de conductas compulsivas, apoyándose en supersticiones que distorsionan la esencia de una religión: la búsqueda de la trascendencia personal y colectiva, y en ocasiones, creyendo en un ser divino, según las respuestas de dichos docentes. También se consideró a la religión como guía moral, y marco de explicación y consuelo que aplica allí donde la ciencia no logra satisfacer las incertidumbres. Se señaló también que el pensamiento mágico forma un cuerpo de conocimientos y prácticas que son rechazadas por las religiones oficiales, un elemento más que permite observar las diferencias entre la magia y religión.

La trascendencia de una religión, la creencia que te da en un ser divino, es la trascendencia en la vida posterior. Es saber qué va a pasar después de que mueres. [...] La fe es una comunicación con un ser divino. Entonces, esa comunicación con un ser divino no tiene nada de mágico. Tiene más bien mucho de concreto, porque lo sientes, porque lo experimentas, porque lo ves cada día en tu actuar [...] Otra esencia de la religión es que muchas personas buscan a la religión como un patrón de conducta moral que te guía a hacer entre lo correcto y lo incorrecto, entre lo bueno y lo malo y que te ayuda a ser mejor persona, llámese de cualquier religión. (María)

Nos permite también tener un recurso para afrontar situaciones que en términos de la propia ciencia y de la realidad no son posibles de afrontar. (Cristina)

Las religiones, tal como yo las entiendo, son unas ideologías organizadas, estructuradas, que tienen elementos que están concentrados y que se entrelazan entre sí. Son un cuerpo de ideas y de doctrinas perfectamente armonizados en líneas generales. Mientras que lo mágico lo entiendo yo más como la presencia de alguna fuerza irracional que no se puede explicar, que no requiere explicación por lo demás, que solamente es accesible a la capacidad de alguien que es el mediador de ello. Así lo vería yo, por lo menos. Dentro de las religiones hay pensamientos mágicos, Por ejemplo, la absolución que te dan, el movimiento de manos, es una cosa mágica asociada a la religión. Yo creo que no hay religión que no tenga pensamiento mágico, pero en principio me parece que son cosas diferentes. (Alberto)

Por el otro lado, los docentes que consideraron equivalentes ambos pensamientos lo hicieron porque en ambos predominan los afectos (temores, deseos, etc.) y en ambos se recurre a elementos sobrenaturales para sus explicaciones.

[...] hay una relación directa entre la creencia en lo sobrenatural y el pensamiento mágico [...], el arte y la religión están basadas en la emoción. (Sergio)

Se preguntó a los participantes por sus impresiones y actitudes hacia aquellos eventos que no logran ser explicados por la psicología estándar, como por ejemplo, las llamadas percepciones extrasensoriales. Dichos eventos son considerados como objeto de estudio de la parapsicología, reconocida por la APA como el estudio sistemático de los fenómenos de transferencia anómala de información o energía (VandenBos, 2006). 
En la mayoría de los casos, los docentes consideraron que los conocimientos de la parapsicología son inválidos e incluso están vinculados con la charlatanería y estafa. En pocos casos se validaron los fenómenos extrasensoriales.

Un pensamiento mágico vendría a ser aquellas personas que creen en lo extrasensorial, que va más allá de los sentidos. [María]

Bueno... la parapsicología existe [...], es la percepción extrasensorial. (Victoria)

Esos son fenómenos parapsicológicos que están siendo explicados por fenómenos perceptivos. (Sara)

Hay elementos parapsicológicos que tienen existencia, pero que como no se repiten con tanta frecuencia, entonces la ciencia no puede sacar leyes científicas. (Cristina)

El tercero de los tres temas abordó el núcleo de la investigación: la presencia e implicancia del pensamiento mágico en la profesión psicológica (figura 3). En la primera categoría se identificó que la mayoría de los docentes validó la distinción entre una psicología científica/académica de una psicología popular.

Yo creo que sí es válida la diferencia [...]. La psicología popular es una psicología intuitiva, es una teoría improvisada, la aprendes sin necesidad de una academia, ni de rigor, ni nada. (David)

Todo el mundo puede hacer psicología [...]. 0 sea, todos nos explicamos. Nos explicamos mágicamente, nos explicamos científicamente, nos explicamos religiosamente. (Sara)

Entre otras respuestas que cuestionaron la demarcación entre psicología científica y no científica, estuvo la siguiente:

[Lo científico] yo creo, para nosotros, es primordialmente basado en una visión occidental, europea, anglosajona, etc. [...] hay un sesgo de poder [risa], de quién tiene el poder, quién determina qué es válido, qué no es válido, quién determina qué es científico y qué no es científico [...], siendo la psicología algo que tiene que ver con el desarrollo humano, con la apreciación del ser, de uno, no nos hemos interesado en la psicología, por ejemplo, de nuestras propias culturas [...], no ha habido mucho interés en, digamos, abrirnos a esas otras maneras de conceptualizar la psicología. (Mónica)

Con respecto a la influencia que el pensamiento mágico podría tener en la formación científica de la psicología, los docentes expresaron que, al ser conocimientos y pensamientos antagónicos, podría ocasionar un conflicto conceptual durante el proceso formativo.

[...] es una limitación. No tienes la flexibilidad suficiente como para abrir el espacio a la ciencia y muchas veces no deseas hacerlo. Sí pienso que obstaculiza. [Cristina]

Ahora, el estudiante que más carga trae de pensamiento mágico le va a ser más difícil aceptar las explicaciones científicas y va a tratar de hacer que coincidan y coordinen [...]. Entonces, inevitablemente va a estar en un estado de conflicto, conflicto conceptual. (César) 


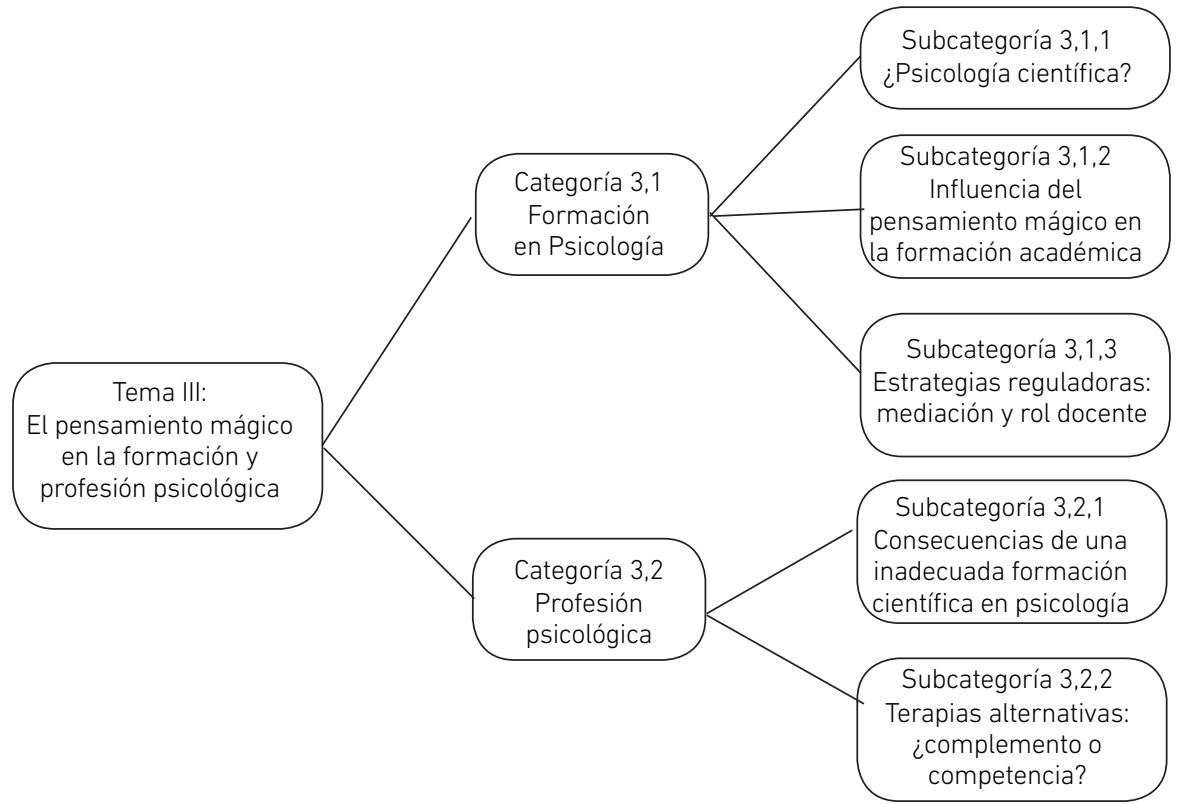

Figura 3. Organización esquemática del tercer tema, sus categorías y subcategorías

Asimismo, algunos entrevistados consideran que el pensamiento mágico debe regularse durante la carrera. Señalaron como opciones (i) el manejo adecuado de los docentes durante las clases, estimulando el pensamiento crítico, y (ii) la importancia de contar con instrumentos adaptados al contexto para medir los niveles de pensamiento mágico.

La segunda categoría examinó las repercusiones del pensamiento mágico en el profesional de psicología.

Lo que a mí me llama la atención es que un psicólogo formado haga brujería o Tarot, o las terapias de regresión. Entonces tú dices: ¿qué pasó con el proceso de formación de ese psicólogo? (Sara)

Según las representaciones de los docentes, una inadecuada formación científica en psicología puede generar perjuicios para el propio psicólogo, para el paciente y para la sociedad, principalmente porque el psicólogo no habrá logrado reemplazar las explicaciones mágicas de la conducta humana por explicaciones científicas, y de esa manera, ya como profesional, va a validar las explicaciones mágicas de las personas en la sociedad. Otra repercusión señalada por la muestra tiene que ver con la relación entre el pensamiento mágico y el locus de control externo ante los eventos. Esa relación disminuye la responsabilidad en las personas, Por lo tanto, cuando el psicólogo brinda explicaciones 
mágicas, fomenta el pensamiento mágico y, en consecuencia, atenta contra la responsabilidad individual y colectiva,

[...] porque si las cosas al final del día dependen de la magia, de Dios, del destino, del chamán... la verdad es que ¿cómo asumimos nuestra responsabilidad y cómo le encontramos causas naturales? Entonces es una abdicación de la responsabilidad [...] porque ya no depende de uno, sino depende de [breve risa] poderes sobrenaturales ¿y qué va a hacer uno frente a poderes sobrenaturales? Nada. Rezar y confiar. (Guillermo)

Se halló también una división entre las consideraciones de los docentes sobre si las terapias alternativas eran un complemento o una competencia para la psicoterapia estándar. Un grupo reclama mayor flexibilidad a los colegas que muestran mucha rigidez por la evidencia científica y, de esa manera, no permiten que los pacientes exploren alternativas acordes con su cosmovisión. Lo que importaría, según ellos, es cuál narrativa deciden creer los pacientes, más que la comprobación de la veracidad.

Aquí estamos entrando al ámbito de que la psicología clínica en particular, lo único que necesita es articular historias coherentes, historias que tengan sentido para la persona que lo está viviendo [...] yo creo que en ese sentido las terapias clínicas no necesitan una teoría de la verdad. (David)

Otros entrevistados manifiestan que las psicoterapias alternativas o complementarias, como las llaman a veces, pueden ayudar en reducir el tiempo de recuperación del paciente, a la vez que se les pueden ir presentando los beneficios de la psicoterapia convencional, pero teniendo cuidado de no «contaminar el método y discurso» de ambas terapias; es decir, no favorecer las explicaciones sobrenaturales ni un locus de control externo. Algunas otras respuestas van en sentido contrario. Hay una competencia entre las terapias con evidencias y aquellas sin evidencias. El principal reclamo es la posibilidad de no ser inocuas o poder interferir con otros tratamientos de eficacia probada (costo de oportunidad).

Finalmente, en el mismo ámbito de la salud, hay respuestas que hacen un llamado a la ética psicológica. Incluso, esperando una pronunciación del Colegio de Psicólogos sobre la validez de permitir psicoterapias alternativas que no gozan de suficiente evidencia científica.

[...] valdría la pena verlo, si es que el Colegio de Psicólogos no debería regular de alguna forma, o sea, si un psicólogo decide utilizar cualquiera de estos tratamientos que no tienen ninguna evidencia científica a favor, el colegio de psicólogos debería normar eso. (Guillermo) 


\section{DISCUSIÓN}

Con respecto al primer tema (aproximaciones y explicaciones a los eventos del mundo), las explicaciones naturales-científicas gozaron de un consenso entre los docentes, siendo sus conceptualizaciones afines a las convencionales (Gauch, 2009). Con respecto a las explicaciones sobrenaturales, si bien no todos los entrevistados manifestaron creer en su ocurrencia, sí hubo consenso en definirla como una explicación que desafía los estándares de la ciencia, estando en concordancia con trabajos que abordan su definición tanto en lo conceptual como en lo operacional (Eckblad y Chapman, 1983; Kingdon, Egan y Rees, 2012; Moral; 2009; Tobacyk, 1984).

Cabe resaltar que tanto los entrevistados como los trabajos hallados en la literatura, definen lo sobrenatural, pero no se aclaran si hay o no diferencias con términos afines. Hacer ese distingo sería de utilidad para estudios en esa línea, pues como han señalado Lindeman y Svedholm, "es probable que la interrelación entre los conceptos sobrenatural, mágico, paranormal y superstición sea similar a la interrelación de los conceptos mente, espíritu y alma. Conceptos que tienen diferentes connotaciones y, aun así denotan la misma cosa (2012, p, 247).

Otro grupo de explicaciones se denominaron intermedias por no ser caracterizadas enteramente como científicas-naturales ni como sobrenaturales. Tanto las explicaciones pseudocientíficas como la concepción dualista-naturalista que presentó un docente, permitieron evidenciar dos cosas. Por un lado, la necesidad de discutir y precisar los matices entre que hay en los límites que separan una explicación natural de una no natural, Por otro, se observó que los docentes que más precisiones ofrecieron en sus respuestas sobre los tipos de explicaciones fueron docentes dedicados a la investigación y al análisis conceptual de su campo, ya sea por su formación o por asignaturas a su cargo. Esta misma observación fue señalada por Otis y Alcock (1982).

Al indagar por una posible jerarquía entre las fuentes del conocimiento y las explicaciones antes descritas, se halló un disenso entre los entrevistados. Se observa que, por un lado, hay un grupo de docentes que priorizan la explicación científica en todo ámbito, y otro grupo que otorga un mismo estatus a las explicaciones mágicas cuando la ciencia muestra una impotencia explicativa, pues de acuerdo a su entendimiento, lo que importa es recobrar el equilibrio interno sea cual sea la explicación que vaya acorde al sistema de creencia de las personas.

Con relación al primer grupo de docentes, se vuelve oportuno mencionar la diferencia entre tecnología y pseudotecnologías. Según Bunge (1980), una tecnología o técnica es producto de conocimientos avalados por la ciencia, y por eso se mostrarían eficaces y útiles para la vida de las personas, como han remarcado algunos entrevistados. 
Asimismo, los conocimientos no avalados por la ciencia desarrollarían pseudotécnicas o pseudotecnologías que pueden no garantizar el bienestar de la sociedad, tópico que se discute más adelante.

Con relación al grupo de docentes que asigna un mismo peso a las explicaciones científicas y mágicas en el ámbito privado, permitiendo así la interpretación de que cualquier explicación es válida mientras genere calma, se presenta a continuación una duda y una posible respuesta debido a lo importante que es este punto para la investigación,

¿Qué proceso subyace en los profesionales de la psicología que, estando comprometidos con los valores y procedimientos de la ciencia, otorgan una misma validez explicativa a una fuente mágica? La posible respuesta se sostiene, por una parte, en lo que Guerrero, Ávila y Miranda (2008) han llamado "hipótesis de una ignorancia selectiva". Esto significa que, en determinados momentos, algunos científicos pueden renunciar a los principios básicos del pensamiento científico para dar lugar a explicaciones mágicas por sus beneficios. ¿Cuáles serían esos determinados momentos y esos beneficios? La otra parte de la respuesta se completa con los aportes de Berembaum, Boden y Baker (2009). Estos autores señalan que ciertos estímulos o eventos tienen un impacto emocional significativo que se acompaña con una baja tolerancia a la incertidumbre y obliga a la persona a intentar conseguir un cierre cognitivo, alguna explicación que reduzca la alteración por la que pasa, y eso lo lleva a cometer sesgos y saltos en sus conclusiones, pudiendo apoyarse en explicaciones irracionales si así fuese necesario.

Pasando al segundo gran tema que se recogió de las entrevistas (el pensamiento mágico y áreas afines), se encuentra un consenso en el modo por el cual los entrevistados entienden lo que implica pensar mágicamente. Sus representaciones están en consonancia con las aproximaciones clásicas, es decir, aquellas que caracterizan al pensamiento mágico como la predominancia de los afectos en los juicios, las asociaciones erróneas, su protagonismo en momentos de incertidumbre y falta de control, la evolución del pensamiento en etapas, y su función de dar sentido a las cosas (Frazer, 2011; Freud, 1913; Lévi-Strauss, 1997; Malinowski, 1948; Piaget, 1929; Tylor, 1920).

Así también, los entrevistados relacionaron el pensamiento mágico con lo sobrenatural, Esta conceptualización se halla en las definiciones modernas (Eckblad y Chapman, 1983; Kingdon, Egan y Rees, 2012; Moral, 2009; Tobacyk, 1984). Algunos de los docentes también consideraron el pensamiento mágico como un rasgo connatural al ser humano, lo que se observa también en trabajos clínicos y experimentales al respecto (Bell, Reddy, Halligan, Kirov y Ellis, 2007; Lindeman et al., 2013; Ramachandran, 2012; Riekki et al., 2014; Te Wildt y Schultz-Venrath, 2004).

Al comparar el pensamiento mágico con el pensamiento religioso, se encontró una nueva falta de consenso, como se observa en los resultados. Quizás lo más relevante para la discusión de ese punto es que los docentes que se clasificaron como religiosos se 
diferenciaron de sus correligionarios, pues puede observarse la distinción que hallaban entre ser religioso y tener un pensamiento mágico. En resumidas cuentas, el docente religioso manifiesta tener un entendimiento más amplio del mensaje trascendental de la religión, de la participación de un ser divino en el mundo, y que ellos no caen en conductas obsesivas-compulsivas como sí lo harían aquellos religiosos con pensamiento mágico o conductas supersticiosas, como señalan. Hallazgos similares encontró Peñaloza (2016) en docentes de ciencia, quienes, profesando una religión, tenían un entendimiento distinto sobre ella y sobre la naturaleza y participación de un ser divino sobre el mundo, en comparación de sus correligionarios no científicos.

Es posible, entonces, considerar que la formación científica en los propios docentes religiosos tiene un efecto en el entendimiento que poseen sobre la religión y lo divino, a diferencia de aquellas personas que no cuentan con una misma formación. Al trasladar este hallazgo al salón universitario, cobra importancia la sugerencia de Glennan (2009) de discutir y definir lo que se entiende por religión, divinidad, fe, etcétera, pues aparte de la delimitación conceptual, cada docente podrá revelar a sus estudiantes los alcances que considera que tiene la ciencia con respecto a la religión. Un ejemplo es la propia distinción que hace Glennan (2009) al señalar que el aspecto religioso y la conexión divina son fenómenos que se reservan a un ámbito privado y escapan del alcance de la ciencia. Otro puede ser el caso en el cual los docentes consideren que el fenómeno religioso puede ser apropiadamente examinado desde una perspectiva científica (Dennett, 2011).

Pasando a las representaciones de los docentes sobre el estudio de los fenómenos anómalos, también llamados parapsicológicos, se observa claramente una actitud escéptica y, en ocasiones, invalidante. Es relevante señalar que dicha mayoría no estaría coincidiendo con el reconocimiento que da la APA al campo que estudia los procesos anómalos de transferencia de información y energía (VandenBos, 2006), pues la considera comprometida con los valores y metodología de la ciencia, incluyendo principalmente el estudio de las percepciones extrasensoriales.

La exploración del tópico paranormal, además de permitir observar cómo los docentes se aproximan a los límites de la ciencia, ofreció otro hallazgo: dos docentes de la muestra, con clara actitud prociencia a lo largo de sus entrevistas (Sara y Cristina), validaron los fenómenos parapsicológicos por experiencia directa y vinculación con trabajos de investigación (en el caso de Sara). Este hallazgo refuerza lo señalado por Miles (2000), quien indicó que el rechazo predominante hacia la parapsicología por los psicólogos guarda relación con la falta de fundamentos y experiencias directas. El trabajo de Miles hace un llamado a la discusión honesta, alturada y fundamentada sobre los avances de dicho campo, de modo que las actitudes de los principales escépticos (psicólogos, como él señala) puedan variar y balancear la desigualdad de recursos (humanos y económicos) que tiene la investigación parapsicológica en comparación con otra. La 
presente investigación rescata de este tópico la importancia de estimular la curiosidad y aproximación científica hacia los campos lindantes de la ciencia estándar. Esto en aras de una honesta búsqueda del conocimiento natural del mundo.

Finalmente, se discute el tercer y último tema (el pensamiento mágico en la formación y profesión psicológica). Si bien los docentes validaron la importancia de diferenciar una psicología popular de una psicología científica, aquella que incorpora el método y conocimientos de otras ciencias, también hubo un reclamo válido. Dicho reclamó señaló el sesgo cultural que hay por una psicología occidentalizada, que etiqueta como científico aquello que va acorde con los estándares de dicha cultura, desestimando e ignorando la psicología de sociedades distintas. Este sesgo, llamado anglocéntrico, hace la tácita equivalencia entre ser humano y cultura occidental y fue criticado también por Moghadam (1987). Aquello pone en relieve la necesidad de seguir considerando los aportes de la psicología transcultural para evitar caer en el sesgo cultural señalado.

Con respecto al pensamiento mágico en la formación científica de psicología, este representa un obstáculo para el pensamiento científico, en principio, por postular explicaciones sobrenaturales que desafían frontalmente las categorías fundamentales de la ciencia (Lindeman y Svedholm, 2012), lo que es contrario al razonamiento científico según la APA (VandenBos, 2006). Luego, porque el pensamiento mágico es un proceso irreflexivo donde los afectos comprometen la interpretación de los eventos (Berenbaum et al., 2009; Freud, 1913; Malinowski, 1948; Piaget, 1929), lo que conlleva a cometer erróneas asociaciones (Frazer, 2011; Tylor, 1920) a la hora de postular explicaciones. Como apuntó Frazer: "una asociación adecuada produce ciencia, pero una inadecuada produce la magia" (2011, p. 38).

Así también, un pensamiento mágico estaría faltando, por lo menos, a dos pilares fundamentales de la ciencia: el uso de (i) principios lógicos donde los enunciados permitan llegar a conclusiones con (ii) evidencias (Gauch, 2009). El pensamiento mágico y sus creencias sobrenaturales comprometen la capacidad crítica de evaluar la información y puede ello interferir en un adecuado aprendizaje en ciencias, y particularmente en psicología, como se ha observado (Bensley, Lilienfeld y Powell, 2014; Messer y Griggs, 1989). Finalmente, un pensamiento crítico debilitado puede abrir las puertas a todo tipo de pseudociencias y pseudotécnicas (Bunge, 1980), lo que no solo pervierte el desarrollo de ciencias incipientes (Bunge, 2000) sino que tiene implicancias éticas cuando se lleva al campo de la salud, como en el caso de la psicología clínica (Lilienfeld, Lynn y Lohr, 2003).

En suma, las representaciones de la mayoría de los docentes (también de los que profesan una religión) evidencian que el pensamiento mágico es un obstáculo para la formación científica en psicología y esperan su reducción durante la carrera, aunque reconocen que no necesariamente sucederá. Así también, ser religioso no es un 
impedimento para formarse científicamente, pues dependerá de cómo se entiendan los campos de acción de la religión, lo divino y la ciencia.

Finalmente, la crítica hacia los psicólogos que validan el pensamiento mágico en su práctica profesional y ofrecen o sugieren terapias alternativas o mágicas cuya eficacia no goce de suficiente encuentra respaldo de la APA y otros autores que esperan que los psicólogos reemplacen las explicaciones populares o mágicas sobre el ser humano por explicaciones científicas (Bunge, 2009; Cárdenas et al., 2013: Lilienfeld et al., 2003; Lilienfeld et al., 2012; VandenBos, 2006).

Entre las consecuencias de validar el pensamiento mágico se señala la estimulación del locus de control externo, cuya relación con el pensamiento mágico está documentada en literatura (Arad, 2014; Bocci y Gordon, 2007; Eremsoy y Inozu, 2016; Farkas, 2003; Hamerman y Morewedge, 2015; Keinan, 2002; Sagone y De Caroli, 2015; Yorulmaz, Inozu y Gultepe, 2011). Por tal motivo, se halló el reclamo de que un mayor locus de control externo amenaza la responsabilidad individual por convertirse en agente de cambio para la sociedad. Así también, puede atentar contra el desarrollo de un pensamiento crítico que permita cuestionar las imposiciones morales de las religiones sobre la sociedad.

\section{CONCLUSIÓN}

La investigación buscó contribuir, a través de una aproximación diferente, con el conjunto de investigaciones interesadas en entender la presencia e influencia del pensamiento mágico en ámbitos académicos de formación científica, dando a conocer las representaciones que tienen los docentes de psicología sobre la influencia del pensamiento mágico en la formación y profesión psicológica.

Las representaciones exploradas permiten observar faltas de consenso que pueden ser perjudiciales para los estudiantes y para la disciplina, al no encontrar consistencia entre sus docentes. Tales inconsistencias giran en torno a la magia y religión, al estudio de las percepciones extrasensoriales, terapias alternativas y convencionales, y todo aquello que puede enmarcarse dentro de los alcances y límites de la psicología como ciencia.

La falta de consenso es llamativa y puede sugerir causas sobre la presencia del pensamiento mágico en estudiantes de psicología, pues si los propios docentes no presentan conceptualizaciones alineadas a una formación científica, es de esperar que sus estudiantes conciban la psicología - y la ciencia en sí- de maneras variadas, e incluso contradictorias.

Entre las variables sociodemográficas, los docentes con un perfil dedicado a la investigación o cuyos cursos ejercitan la reflexión filosófica o el análisis conceptual presentaron más observaciones y precisiones a lo largo de la entrevista. Estas variables 
se encontraron más relacionadas con los aportes sobre el tema, a diferencia del sexo, edad y años de docencia.

Finalmente, la falta de consenso permite identificar la necesidad de crear espacios donde los docentes de psicología discutan y revisen contenidos sobre la filosofía de la ciencia y de la psicología, de modo tal que la disciplina se fortalezca al tener un cuerpo docente cada vez más unificado en sus representaciones en torno a la problemática aquí estudiada.

\section{REFERENCIAS}

Arad, A. (2014). Avoiding greedy behavior in situations of uncertainty: the role of magical thinking. Journal of Behavioral and Experimental Economics, 53, 17-23. https://doi. org/10.1016/j.socec.2014.07.003

Ávila, A. (2001). Los profesores y sus representaciones sobre la reforma de las matemáticas. Perfiles educativos, 23(93), 59-86.

Bell, V., Reddy, V., Halligan, P., Kirov, G., y Ellis, H. (2007). Relative suppression of magical thinking: A Transcranial Magnetic Stimulation Study. Cortex, 43, 551-557. https:// doi.org/10.1016/S0010-9452(08)70249-1

Bensley, D., Lilienfeld, S. y Powell, L. (2014). A new measure of psychological misconceptions: Relations with academic background, critical thinking, and acceptance of paranormal and pseudocientfic claims. Learning and Individual Differences, 36, 9-18. https://doi.org/10.1016/j.lindif.2014.07.009

Berenbaum, M., Boden, T. y Baker, J. (2009). Emotional salience, emotional awareness, peculiar beliefs and magical thinking. Emotion, 9(2), 197-205. doi: 10.1037/ a0015395

Bocci, L y Gordon, K. (2007). Does magical thinking produce neutralising behaviour? An experimental investigation. Behaviour Research and Therapy, 45, 1823-1833. https://doi.org/10.1016/j.brat.2007.02.003

Bunge, M. (1980). Epistemología, curso de actualización. Barcelona: Ariel.

Bunge, M. (2000). La investigación científica; su estrategia y filosofía. México, D. F.: Siglo xxı.

Caldera, J., Amador, G., Reynoso, O. y Zamora, M. (2015). Pensamiento mágico en estudiantes de psicología. Un estudio descriptivo y correlacional. Revista de educación y desarrollo, 35, 29-36. Recuperado de https://www.researchgate. net/profile/Juan_Montes8/publication/295102934_Pensamiento_magico_ en_estudiantes_de_Psicologia_Un_estudio_descriptivo_y_correlacional/ links/57fd572b08ae406ad1f3d0db/Pensamiento-magico-en-estudiantes-dePsicologia-Un-estudio-descriptivo-y-correlacional.pdf 
Caldera, J., Reynoso, O., Zamora, M. y Pérez, I. (2017). Pensamiento mágico en estudiantes. Estudio comparado entre niveles educativos. Revista Iberoamericana para la Investigación y el Desarrollo Educativo, 8(15), 1-25. doi: http://dx.doi.org/10.23913/ ride.v8i15.308

Cárdenas, M., Gallardo, I., Adaos, R. y Bahamondes, J. (2013). Creencias paranormales en una muestra de estudiantes de psicología en universidades chilenas. Salud y Sociedad, 4 (1), 10-23. https://doi.org/10.22199/S07187475.2013.0001.00001

Castaño, C. y Quecedo, R. (2002). Introducción a la metodología cualitativa. Revista de Psicodidáctica, 14, 5-39. Recuperado de http://www.redalyc.org/pdf/175 /17501402.pdf

Covarrubias, P. (2016). Representaciones docentes de la educación basada en competencias. Un estudio de caso. Propósitos y Representaciones, 4(2), 73-132. http://dx.doi.org/10.20511/pyr2016.v4n2.120

Damn, J. (2014). Representaciones y actitudes del profesorado frente a la integración de niños/as con necesidades educativas especiales al aula común. Revista Latinoamericana de Educación Inclusiva, 25-35. Recuperado de http:// www.repositoriocdpd.net:8080/bitstream/handle/123456789/78/Art_ DammMunozX_RepresentacionesActitudesProfesorado_2008.pdf?sequence=1

Declaración de Budapest (1999). Declaración sobre la ciencia y la utilización del conocimiento científico [versión electrónica]. Recuperado de http://www.encuentros-mul tidisciplinares.org/Revistan\%C2\%BA2/Declaraci\%C3\%B3n\%20sobre\%20 ciencia.pdf

Dennett, D. (2011). Romper el hechizo. La religión como fenómeno natural. Madrid: Katz.

Denzin, N. y Lincoln, Y. (2012). Manual de investigación cualitativa. Barcelona: Gedisa.

Dudycha, G. (1933). The superstitious beliefs of college students. The Journal of Abnormal and Social Psychology, 27(4), 457-464. http://dx.doi.org/10.1037/h0069831

Eckblad, M. y Chapman, L. (1983). Magical ideation as an indicator of schizotypy. Journal of Consulting and Clinical Psychology, 51(2), 215-225. http://dx.doi. org/10.1037/0022-006X.51.2.215

Eremsoy, E. y Inozu, M. (2016). The role of magical thinking, religiosity and thought control strategies in obsessive-compulsive symptoms in a turkish adult sample. Behaviour Change, 33(1), 1-14. https://doi.org/10.1017/bec.2015.16

Farkas, C. (2003). Utilización de estrategias mágicas para el manejo de situaciones estresantes en estudiantes universitarios. Revista Interamericana de Psicología, 37(1), 109-143. doi: http://dx.doi.org/10.30849/rip/ijp.v37i1.811

Flick, U. (2015). El diseño de investigación cualitativa. Madrid: Morata. 
Fraile, C., Prieto, L. y Bacaicoa, F. (2005). Las representaciones de la tutoría universitaria en profesores y estudiantes: estudio de un caso. International Journal of Psychology and Psychological Therapy, 5(2), 148-168. Recuperado de http://www. redalyc.org/html/560/56050205/

Frazer, J. (2011). La rama dorada (O. Figueroa Castro, Trad.). México, D. F: Fondo de Cultura Económica (trabajo original publicado en 1890).

Freud, S. (1913). Tótem y tabú y otras obras [versión de Adobe Acrobat Reader]. Recuperado de http://www.bibliopsi.org/docs/freud/13\%20-\%20Tomo\%20XIII.pdf

Gauch, H. (2009). Science, Worldviews, and Education. Science \& Education, 18(6), 667-695. doi 10.1007/s11191-006-9059-1

Guerrero, C., Ávila, R. y Miranda, P. (2008). La correlación entre creencias mágicas y variables sociodemográficas. Psicología y Ciencia Social, 10(1-2), 5-15. Recuperado de https://scholar.google.com/scholar_url?url=http://tuxchi.iztacala.unam.mx/ ojs/index.php/pycs/article/download/12/10\&hl=es\&sa=T\&oi=gsb-ggp\&ct=res\& $\mathrm{cd}=0 \&$ ei=MAPeWsykM4memgHyqbLYCw\&scisig=AAGBfm0GYv1KyjGaPWetqDk ZGwyds7pkyw

Hamerman, E. y Morewedge, C. (2015). Reliance on luck: Identifying which achievement goals elicit superstitious behavior. Personality and Social Psychology Bulletin, 4(3), 323-335. doi: 10.1177/0146167214565055

Hernández, S., Fernández, C. y Baptista, P. (2014). Metodología de la investigación. México, D. F.: McGraw-Hill.

Jahoda, G. (1968). Scientific training and the persistence of traditional beliefs among west African university students. Nature, 220, 1356. doi:10.1038/2201356a0

Keinan, G. (2002). The effects of stress and desire for control on superstitious behavior. Personality and Social Psychology Bulletin, 28, 102-108. doi: $10.1177 / 0146167202281009$

Kingdon, B., Egan, S. y Rees, C. (2012). The Illusory Beliefs Inventory: A new measure of magical thinking and its relationship with obsessive compulsive disorder. Behavioural and Cognitive Psychotherapy, 40, 39-53. https://doi.org/10.1017/ S1352465811000245

Kvale, S. (2011). Las entrevistas en investigación cualitativa. Madrid: Morata.

Lévi-Strauss, C. (1997). El pensamiento salvaje [versión Adobe Acrobat Reader]. Recuperado de https://monoskop.org/images/1/18/Levi-Strauss_Claude_El_ pensamiento_salvaje_1997.pdf

Lilienfeld, S., Lynn, S. y Lohr, J. (eds.) (2003). Science and pseudoscience in clinical psychology. New York: Guilford. 
Lindeman, M., Svedholm, A., Riekki, T., Raij, T. y Hari, R. (2013). Is it just a brick wall or a sign from the universe An fMRI study of supernatural believers and skeptics. Social Cognitive and Affective Neuroscience, 8(8), 943-949. https://doi.org/10.1093/ scan/nss096

Lindeman, M. y Svedholm, A. (2012). What's in a term? Paranormal, superstitious, magical and supernatural beliefs by any other name would mean the same. Review of General Psychology, 16(3), 241-255. doi: 10.1037/a0027158

Malinowski, B. (1948). Magia, ciencia y religión [versión Adobe Acrobat Reader]. Recuperado de https://asodea.files.wordpress.com/2009/09/malinowski-bronislaw-magiaciencia-y-religion.pdf

Martínez-Salgado, C. (2011). El muestreo en investigación cualitativa. Principios básicos y algunas controversias. Ciencia \& Saude Coletiva, 17(3), 613-619. doi: 10.1590/ s1413-81232012000300006

Mazzitelli, C., Aguilar, S., Guirao, A. y Olivera, A. (2009). Representaciones sociales de los profesores sobre la docencia: contenido y estructura. Educación, lenguaje y sociedad, 6 (6), 265-290. Recuperado de http://s3.amazonaws.com/ academia.edu.documents/31068664/n06a14mazzitelli.pdf?AWSAccessKeyld $=$ AKIAIWOWYYGZ2Y53UL3A\&Expires $=1499835354 \&$ Signature $=R 2 z T O K D u g R$ \%2F0kS6z09ywSZjvEpM\%3D\&response-content-disposition=inline\%3B\%20filename\%3DRepresentaciones_sociales_de_los_profeso.pdf

McCaffree, K. y Saide, A. (2014). Why is critical thinking so hard to teach? Skeptic, 19(4), 54-64. Recuperado de http://go.galegroup.com/ps/anonymous?id=GALE\%7CA3 94997149\&sid=googleScholar\&v=2.1\&it=r\&linkaccess=fulltext\&issn=10639330 $\& p=A O N E \& s w=w \&$ authCount $=1 \&$ isAnonymousEntry=true

Messer, W. y Griggs, R. (1989). Student belief and involvement in the paranormal and performance in introductory psychology. Teaching of Psychology, 16(4), 187-191. Recuperado de http://journals.sagepub.com/doi/abs/10.1207/s15328 023top1604_4

Miles, R. (2000). A Study of Psychologists' Attitudes Toward Psi (tesis doctoral). Antioch New England Graduate School. New Hampshire.

Moghaddam, F. (1987). Psychology in the three worlds. As reflected by the crisis in social psychology and the move toward indigenous third-world psychology. American Psychologist, 42(10), 912-920. Recuperado de http://fathalimoghaddam.com/ wp-content/uploads/2013/10/1258685560.pdf

Molina, S. (2003). Representaciones mentales del profesorado con respecto al fracaso escolar. Revista Interuniversitaria de Formación del Profesorado, 17(1), 151-175. Recuperado de http://www.redalyc.org/html/274/27417110/ 
Moral, J. (2009). Escala para medir superstición y pensamiento mágico. México, D. F.: Psicom.

Moscovici, S. (1993). Psicología Social, II. Pensamiento y vida social. Psicología social y problemas sociales. Barcelona: Paidós.

Otis, L. y Alcock, J. (1982). Factors affecting extraordinary belief. The Journal of Social Psychology, 118, 77-85. doi.org/10.1080/00224545.1982.9924420

Otis, L. y Kuo, E. (1984). Extraordinary beliefs among students in Singapore and Canada. The Journal of Psychology: Interdisciplinary and Applied, 116(2), 215-226. doi: $10.1080 / 00223980.1984 .9923639$

Peñaloza, G. (2016). Una exploración de las ideas sobre las relaciones entre religión y ciencia de profesores de ciencias naturales. Revista Nures, 14(34), 1-13. Recuperado de https://revistas.pucsp.br/index.php/nures/article/view/32165

Petra, I. y Estrada, A. (2014). El pensamiento mágico: diseño y validación de un instrumento. Investigación en Educación Médica, 3(9), 28-33. Recuperado de http://www.scielo. org.mx/scielo.php?pid=S2007-50572014000100005\&script=sci_arttext

Piaget, J. (1929). The Child's Conception of the World [versión Adobe Acrobat Reader]. Recuperado de https://archive.org/details/childsconception01piag

Ramachandran, V. (2012). Lo que el cerebro nos dice. Los misterios de la mente humana al descubierto (J. Soler Chic, trad.). Madrid: Paidós.

Regal, A. (1993). Pensamiento mágico-religioso y pensamiento científico. Humanitas, 26, 13-22.

Riekki, T., Lindeman, M. y Raij, T. (2014). Supernatural believers attribute more intentions to random movement than skeptics: An fMRI study. Social Neuroscience, 9, 400-411. https://dx.doi.org/10.1080/17470919.2014.906366

Robles, B. (2011). La entrevista a profundidad: una técnica útil dentro del campo antropofísico. Cuicuilco, 18(52). Recuperado de http://www.redalyc.org/pdf/351 /35124304004.pdf

Rudski, J. y Edwards, A. (2007). Malinowski goes to college: factors influencing students use of ritual and superstition. The Journal of General Psychology, 134(4), 389-403. https://doi.org/10.3200/GENP.134.4.389-404

Sagone, E. y De Caroli, M. (2015). Beliefs about superstition and luck in external believers university students. Procedia Social and Behavioral Sciences, 19, 366-371. doi: 10.1016/j.sbspro.2015.04.685

Salter, C. y Routledge, L. (1971). Supernatural beliefs among graduate students at the university of Pennsylvania. Nature, 232, 278-279. doi: 10.1038/232278a0

Silva Santisteban, F. (1975). Pensamiento mágico en la Universidad de Lima. Scientia et praxis, 9, 116-146. 
Starks, H. y Brown, S. (2007). Choose Your Method: A Comparison of Phenomenology, Discourse Analysis, and Grounded Theory. Qualitative Health Research, 17(10). 1372-1380. doi: 10.1177/1049732307307031

Te Wildt, B. y Schultz-Venrath, U. (2004). Magical ideation - Defense mechanism or neuropathology?Psychopathology,37,141-144.https://doi.org/10.1159/000078866

Tobacyk, J. (1984). Paranormal belief and college grade point average. Psychological Reports, 54 (1), 217-218. https://doi.org/10.2466/pr0.1984.54.1.217

Travé, G. (2013). Un estudio sobre las representaciones del profesorado de educación primaria acerca de la enseñanza bilingüe. Revista de Educación, 361, 379-402. doi: 10.4438/1988-592X-RE-2011-361-149

Tylor, E. (1920). Primitive Culture. Researches into the development of mythology, philosophy, religion language, art, and custom [versión electrónica]. Recuperado de https://ia902205.us.archive.org/32/items/primitiveculture01tylouoft/primiti veculture01tylouoft.pdf

Tupper, V. y Williams, R. (1986). Unsubstantiated beliefs among beginning psychology students. Psychological Reports, 58, 383-388. Recuperado de http://journals.sage pub.com/doi/pdf/10.2466/pr0.1986.58.2.383

Unesco (2016). Educación científica. Recuperado de http://www.unesco.org/fileadmin/ MULTIMEDIA/FIELD/Montevideo/pdf/PolicyPapersCILAC-CienciaEducacion.pdf

VandenBos, R. y American Psychological Association (2006). American Psychological Association Dictionary of Psychology. Washington, D. C.: American Psychological Association.

Valentine, W. (1936). Common misconceptions of college students. Journal of Applied Psychology, 20(6), 633-658. http://dx.doi.org/10.1037/h0053524

Wagner, M. (1928). Superstitions and their social and psychological correlatives among college students. The Journal of Educational Sociology, 2(1), 26-36. doi: $10.2307 / 2961760$

Willig, C. (2013). Introducing qualitative research in psychology (3. ${ }^{\circ}$ ed.). London: McGrawHill Education.

Yorulmaz, O., Inozu, M. y Gültepe, B. (2011). The role of magical thinking in obsessivecompulsive disorder symptoms and cognitions in an analogue sample. Journal of Behaviour Therapy and Experimental Psychiatry, 42, 198-203. https://doi. org/10.1016/j.jbtep.2010.11.007 
